\title{
Robotic Fabrication of Sustainable Hybrid Formwork with Clay and Foam for Concrete Casting
}

\author{
Weishun Xu \\ Zhejiang University | China | xuweishun@zju.edu.cn \\ Zixun Huang \\ Zhejiang University | China | 31501012764@zju.edu.cn
}

\begin{abstract}
This paper presents a hybrid formwork fabrication method utilizing additive manufacturing with clay on top of curved foam surfaces robotically fabricated with hot wire. The primary focus of this study is to develop a relatively efficient and highly sustainable formwork manufacturing method capable of producing geometrically complex modular concrete building components. The method leverages fluidity and recyclability of clay to produce uniquely shaped, free-form parts of the mold, and reduces overall production time by using foam for shared mold support/enclosure. A Calibration and tool path generating method based on computational modeling to integrate the two systems are also subsequently developed.
\end{abstract}

Keywords: Robotic fabrication, Hybrid formwork, Mass customization, Clay printing, Foam cutting

\section{INTRODUCTION: EXISTING CHALLENGES OF FORMWORK FABRICATION}

As computational design techniques and the concept of mass-customization challenges the norms of conventional building modules in architecture to accommodate more geometry freedom and variations (Paoletti, 2017), one persisting challenge faced with the off-site fabrication of unique concrete building components with complex shapes has been the manufacturing of formwork. Currently, non-standard formwork fabrication methods widely adopted in completed buildings include CNC milling and hand crafting, of which the cost and sustainability are often the limiting factors due to their long manufacturing time, material waste during fabrication and low mold reusability (Martins \& Sousa, 2014; Schipper, 2015; Timothy et al., 2016).

Recent studies in computer aided manufacturing (CAM) have incorporated novel robotic fabrication systems for potential alternatives to the production of non-standard modular concrete formwork, mostly including hot-blade cutting, reconfigurable molds and additive manufacturing methods (Bell, Read, Ede, \& Barnes, 2014; Martins \& Sousa, 2014; Timothy et al., 2016). However, a large body of studies thus far have proposed fabrication methods for the improvement on either one or two of the main challenges, namely time efficiency, geometrical freedom and sustainability respectively; yet few solution has been proposed and tested to address the fabrication process as a versatile, comprehensive workflow.

An abundant number of existing researches have developed robotic hot wire cutting (RHWC) techniques for modular formwork fabrication out of foam, and such researches demonstrate that the technology may significantly reduce production time as well as wasted material generated compared to conventional CNC milling applied to the same material (Jovanović, Raković, Tepavčević, Borovac, \& Nikolić, 2017; Martins, de Campos, Nunes, \& Sousa, 2018; Søndergaard et al., 2016; Stavric \& Kaftan, 2012). However, most researches show limited capabilities in regard to the level of geometrical freedom of fabricatable building components outside the range of variations and/or combinations of ruled surfaces applied to the entire cut surface, and local details are difficult to execute. The capability to fabricate local changes to modular surfaces also seem to be limited. Meanwhile, material sustainability of RHWC fabrication is still questionable: most researches and projects in this category use expandable polystyrene foam (EPS foam), and currently EPS foam recycling is possible yet hardly practiced. Research efforts in further reducing material waste such as using reciprocal surfaces may introduce more limitations to the design geometry (Jovanovic, Vucic, Stulic, \& Petrovic, 2019).

Research in reconfigurable molds recognizes the need for material efficiency and ecological awareness in the process of formwork fabrication (Kontovourkis, Phocas, \& Katsambas, 2019). However, in the case of modular building components, there appears to be a contracdiction between planar and sectional geometric freedom in existing refonfigurative molds. Currently, systems using flexible angles (Khabazi \& Budig, 2016; Kontovourkis et al., 2019) are unlikely to be merged with systems with pin systems with elastic support surfaces (Bell et al., 2014; Schipper \& Grünewald, 2014). The design solution space associated to the current stage of reconfigurable molds is much confined by the types of actuators. In addition, the lack of flexibility in the molding system itself often leads to a one-off use of the entire design.

Additive manufacturing (3D Printing) in general offers a higher level of geometric freedom to designers, and can be used to process recyclable materials such as clay and wax, both of which can be used for concrete formwork. The 
accuracy of wax printing at the scale of building component is currently unresolved due to limited consistency control of the viscosity of melted wax, causing the resulting formwork to be subject to CNC milling as a post processing procedure, thus significantly reducing its overall fabrication efficiency(Gardiner \& Janssen, 2014). On the other hand, the emerging method of fabricating recyclable formwork with $3 \mathrm{D}$ clay printing (or $3 \mathrm{DCP}$ ) provides the possibility of geometric freedom in design while operating at reasonable efficiency(S. Wang, Dritsas, Morel, \& Ho, 2017). The fact that clay molds contract more than concrete during the dehydration and curing process makes de-molding from complex geometries much easier than many other formwork material choices. However, the accuracy of the system decreases significantly as the horizontal dimension of the formwork increases due to deformation under internal stress and gravity (Carvalho, 2019; Seibold et al., 2018; Sihan Wang, Xuereb Conti, \& Raspall, 2019), rendering the technique challenging to be applied to building component scale at its current stage.

An overview of emerging robotic fabrication methods applied to the production of concrete formwork of modular building components reveals that the issue of overall efficiency conerning material sustainability, geometric freedom and production time still presents critical challenge to individual techniques at current stage. It appears drawbacks of each method may be complemented by the advantage of others under different scales and conditions. Surprisingly, few literature investigates the feasibility of integrating different robitic fabrication techiniques to produce sustainable hybrid formwork for modular building components. In addition, how different robotic fabrication processes can respond to the tolerance of one another through feedback and computational modeling should also be examined.

\section{FABRICATION SYSTEMS AND WORKFLOW OVERVIEW}

The formwork manufacturing method developed by this research integrates two of the reviewed robotic fabrication methods, namely robotic hot wire cutting (RHWC) foam and additive manufacturing with clay (3DCP), in order to efficiently fabricate geometrically complex hybrid formwork for modular architectural geometries while achieving material sustainability in both fabrication and de-molding procedures (Figure 1).

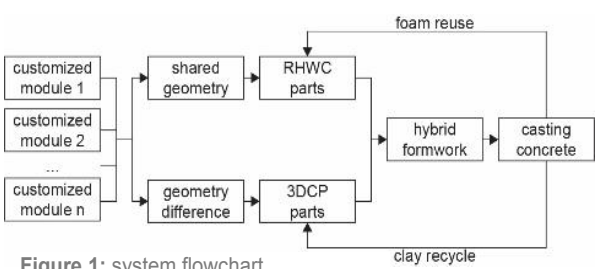

Figure 1: system flowchart

clay recycle

According to our method, curved EPS foam surface forms the basis and boundary of the formwork, spanning across the entire length and width of the building component module. Its curved surface geometry should approximate but not necessarily match the boundary surface of the module; instead, the foam surface should address the maximum amount of volume shared by the negative geometries to all building modules that the framework is intended to be re-used on.
Smaller scale and unique geometrical details in masscustomized modules are materialized by applying 3DCP on top of curved foam surface. Positioning of the foam surface can be adjusted to allow for the maximum level of verticality for the 3DCP parts, in order to minimize its deformation during the dehydration and curing process under gravity. Meanwhile, as a flexible material of decent fluidity, 3DCP also accommodates the inaccuracy in RHWC foam caused by friction between the wire and EPS foam (Figure 2).

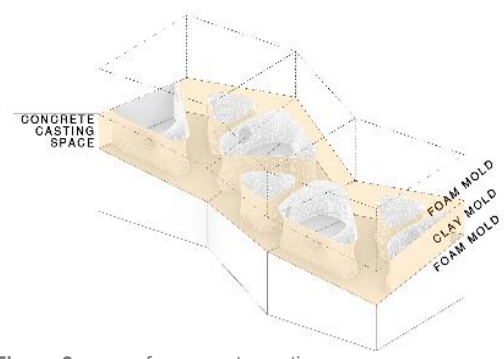

Figure 2: space for concrete casting.

The method takes advantage of the fabrication speed and horizontal span of RHWC foam to siginificantly increase fabrication speed compared to CNC milling, while addressing additional freedom and accuracy in fabricable geometry through the integration of local 3DCP. Maximal reusability of EPS foam is achieved by limiting the use of the material to form only the commonly shared part of all molds for the serial geometries, and 3DCP parts are almost $100 \%$ recyclable after de-molding once dissolved in water.

According to the above-mentioned principles, the digital fabrication tools and subsequent computational tool path generation/calibration mechanisms are thus developed as follows.

\section{HOT WIRE FOAM CUTTING SYSTEM}

The RHWC system (Figure 3) fabricates the foam base surface for the hybrid formwork, which approximates the surface boundary of desired complex module geometry. Considering that local details and imperfections in fabrication can be post-processed by 3DCP, the RHWC system uses a double-arm setup with two KUKA KR120 R2500 industrial robots in order to best preserve the quality of the surface. The double-arm setting can apply bending moments to the cutting wire, allowing for the possibility of forming curved instead of straight wire profiles, in order to fabricate smooth doubly-curved surfaces (Brander et al., 2016). The base frames of the two industrial robots are located 3, 200 millimeters apart (measured center to center) from each other, with a mechanically controlled movable fabrication table of 1,200 millimeter in width and 420 millimeters in height in-between, forming the main platform of which materials sit on. The movable table is 5,000 millimeter in length, and can slide perpendicular to the center-center line between the two industrial robots, effectively extending the range of fabrication by another 2,200 millimeters, thus ensuring the possibility of fabricating large building component scale objects.

For the end effector design, a type of common copper wire with 1 millimeter diameter is chosen as the cutting wire, for which the linear thermal expansion is previously tested by the research team to best ensure control over geometry. 
The ends of the wire are attached through stainless steel clamps to the flanges of the robotic arms, which in turn controls the positions and angles of the last 20 millimeters of the wire. An algorithm is developed so that the flanges turn as the cutting process proceeds in order to ensure that the cutting wire stays in a plane that is perpendicular to the proceeding direction of the cut. The ends of the wire are connected to the positive and negative terminals of a separate switching mode power supply, which pre-heats the wire to a temperature of $400^{\circ} \mathrm{C}$ to subsequently melt the foam.

\section{CLAY EXTRUSION SYSTEM}

The 3DCP system (Figure 3) uses the same industrial robots and movable fabrication table motion system as the RHWC system. Instead of CNC machines based on Cartesian coordinates, 3DCP with a six-axis motion system is perferred due to the needs of printing onto curved surfaces. For this task, it is necessary to ensure that the tip of the material extrusion tool always stays perpendicular to the printing base surface as well as the material formed by previously extruded material in order to minimize deformation. While one of the industrial robots performs $3 \mathrm{DCP}$, the other is programmed as a material-filling machine with a fixed piston as its end effector. The two industrial robots thus work separately on different tasks to ensure highest overall efficiency.

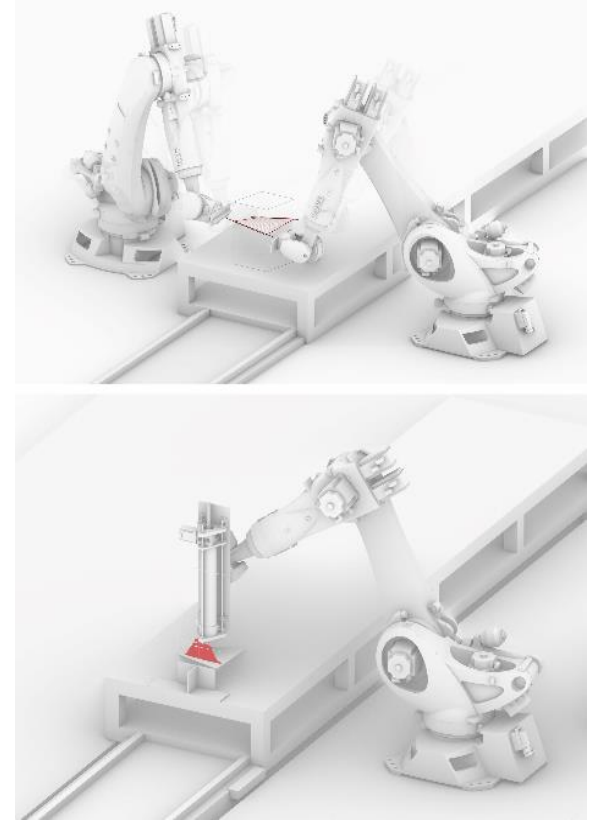

Figure 3: above: robotic hot-wire cutting system; below: robotic $3 \mathrm{D}$ clay printing system

The extrusion end effector consists of four main parts: a step motor (86BYGH250C, $\left.8.5 \mathrm{~N}^{*} \mathrm{M}\right)$ - gear box (1:40 speed reduction) set with piston controlled by a separate DC power supply and stepper drive as the propeller, a stainless steel pipe with a diameter of 100 millimeter and length of 550 millimeter as the material tank, a PLA printed cone-shaped cap with adjustable nozzle attached to its tip as the printing head, and a frame made out of wood plank and stainless steel plates to hold the system in place and attach it to the flange of the robotic arm.

Due to this system setup, the pathing of the end effector and the extrusion process are controlled by the robot server and stepper drive respectively, and need to be synced through a lap top that is connected to both systems.

\section{FROM COMPUTATIONAL MODEL TO FABRICATION: THE WORKFLOW}

Conventional robotic fabrication methods commonly seek to directly translate geometries into tool paths, while the hybrid formwork method uses a responsive computational model in order to calibrate RHWC foam surfaces to prepare for 3DCP. Thus, a formwork design - computational model - RHWC - calibration - 3DCP - casting workflow is developed to allow for systematic control over accuracy.

1. Hybrid formwork design: After extracting the negative geometries from the desired building component modules, the framework design should simultaneously address the characteristics of each robotic fabrication method as well as those of concrete casting and demolding. To ensure maximal reusability of EPS foam, the portion of shared negative geometry by modular building components fabricated in the same batch is extracted by a bool computation in order to inform the design and fabrication of the supporting RHWC foam surface. This operation will determine which part should be fabricated by RHWC and 3DCP respectively. Then, the positioning of formwork for 3DCP fabrication verticality for the clay parts to minimize deformation. This may subsequently decide how the rest of the foam surfaces should be cut.

2. Computational (re)modeling: Since error in the fabrication of RHWC foam is inevitable due to unpredictable friction between foam and wire, and that 3DCP requires accurate base surface data for tool path generation, it is important to re-model the formwork based on computational surfaces tailored to calibration procedures, which should re-generate the model based on measured data from the result of the RHWC foam. The best practice should be to model the RHWC foam surface as one or the combination of a limited number of computational geometries, and several feature points on the surface should be clearly identifiable to serve as input parameters for the computational model (Figure 4). 3DCP parts should be re-modeled as responsive geometries that can be regenerated based on the RHWC surface data.

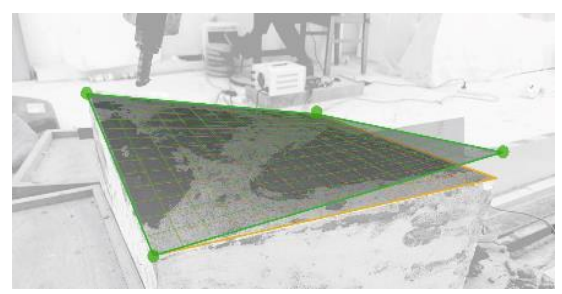

Figure 4: green surface: responsive computational surface, green points: feature points of the computational surface, yellow surface: actual foam surface. 
3. RHWC foam fabrication: A physics simulation-based geometry rationalization strategy is adopted to generate the tooling path for RHWC. The computational surface to be fabricated is sampled as a series of contour curves perpendicular to the direction of the wire movement. The tangent values at the end of the contour curves inform the physics simulation engine to compute the tool path and wire length needed, taking thermal expansion of the heated wire into consideration. Copper wire of matching length is then cut and attached to the robotic arm flanges to execute the fabrication process. In case of calibration points become un-idetifiable due to fabrication inaccuracy, the positioning of modular foam blocks should be clearly marked on the fabrication table, so that RHWC, calibration and 3DCP procedures can share the same physical reference.

4. Calibration: The purpose for calibration is to digitally re-construct the actual surface resulting from RHWC fabrication, in order to generate the base surface upon which 3DCP tool path is decided. Therefore, 3DCP end effector is chosen as the calibration tool, and the tooling procedure should happen before calibration process begins. The tip of the 3DCP extruder is moved under manual operation mode of the industrial robots to sample the spatial positioning of each feature point, and the coordinates are used to digitally regenerate the parametric model of the formwork geometry.
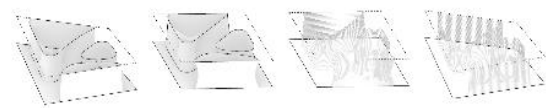

Figure 5: a reciprocal mapping for path generation.

5. 3DCP fabrication: The generation of printing path adopts the common layer-by-layer stacking logic. To compute the tool path on the curved surface, a reciprocal spatial mapping strategy (Figure 5) is programmed to first map desired geometry onto a plane to be contoured at an equal distance, and the contour lines are subsequently mapped back to the calibrated foam surface. Distance between contours depends on the inclination of the printed geometry: layer thickness should be small enough to ensure enough overlapping, but expanded wherever possible to increase printing efficiency. Mold thickness is doubled where higher lateral pressure from concrete casting is expected. After printing, the entire hybrid formwork component is horizontally removed from the fabrication table for clay to cure.
6. Casting and de-molding: The 3DCP parts are let cure before casting, but curing duration needs to be controlled because dry clay mold will cause uneven dehydration of the concrete to produce local cracks. Form release agent is applied to foam surfaces but not necessarily 3DCP parts. Surface texture can be applied to 3DCP parts at the early stage if desired. For concrete mixture, heavy and coarse aggregates are to be avoided, and higher fluidity mixture should be tested if the geometry is complicated.

After concrete cures to a desired level of hardness, the EPS foam bases are demolded first, as further ventilation helps clay to dry and thus initiates the self demolding process. For complex geometries with hard-to-reach spaces, water can be used to dissolve the remaining clay. All materials demolded, including foam and clay, can be re-used either directly for the next module or easily recycled by a pug mill.

\section{PROTOTYPE TEST}

The feasibility of the proposed fabrication method was examined through building a series prototypical concrete exterior wall blocks with unique non-standard form, where foam and clay can complement each other in their geometrical capabilities at different scales. The test blocks were designed as a part of a self-supporting ventilation screen of a model office building southern China (Figure 6). Such building components are often seen in this area as part of the primary climate management system.

The bounding geometry of each block is an extrusion from an equilateral hyperbolic paraboloid (hypar) surface of the same size with side length of 315 millimeters and thickness of 100 millimeters. The size of the block prototype is chosen empirically to allow for local construction workers to easily stack once cast out of concrete. In addition, there are unqiue freeform openings of different sizes and shapes on each wall block to regulate direct sunlight and ventilation based on its position on the facade. To further test the capabilities of clay mold in materializing casting details, a tiny branch of no more than 30 millimeters in diameter was added to articulate the openings.

\section{MOLD DESIGN AND MODELING}

In this design, the hypar surface repeated in all building blocks naturally defines the maximum volume of negative space shared by all modules, and thus forms the targeted surface for RHWC fabrication. The unique fenestration subsequently defines the 3DCP parts. In addition, five pieces of hand-crafted Medium-density fibreboards (MDF)
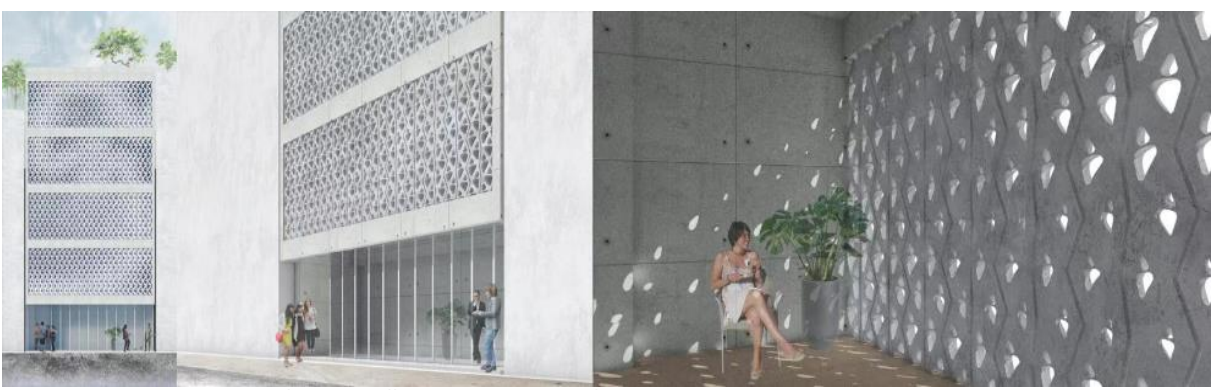

Figure 6: a ventilation screen of a model office building southern China 
were used to help frame and support the hybrid mold (Figure 7).

In the computational model for tool path generating and calibration, the four corners of the hypar surface were chosen as the feature points and thus initial inputs to parametrically create the model for the entire hybrid mold.

Six building blocks were selected to be built at full scale to test the feasibility of the robotic fabrication and mold re-use workflow. To minimize material waste, the bounding hypar surfaces were classified into two types: one is a saddle shape, and the other its complementary geometry. Thus, one hot wire cut could simultaneously create two curvedfoam bases (Figure 8). As a result, we designed and then produced two different hybrid molds for casting.

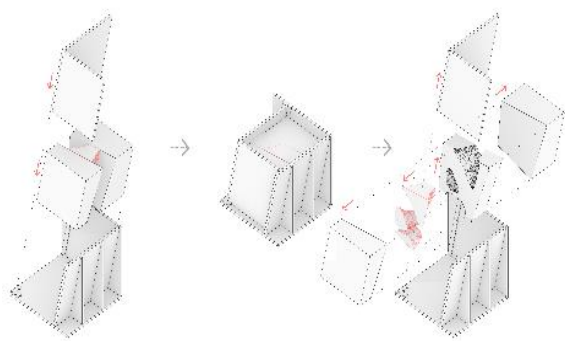

Figure 7: assembly of entire hybrid mold in demonstration.

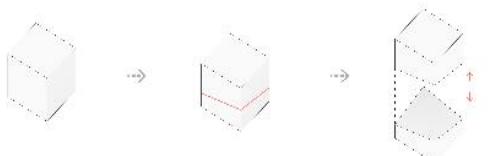

Figure 8: two complementary curved foam bases.

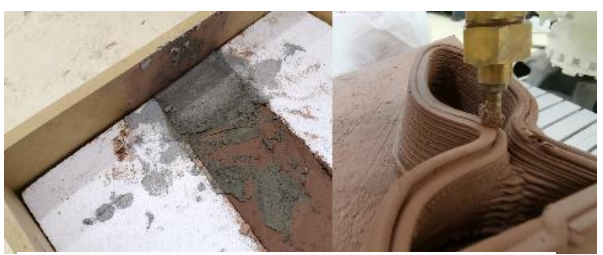

Figure 9: right: double-layered path; left: upturned opening for casting.

\section{ROBOTIC FABRICATION}

Through test cuts on scrap materials, the current on the hot wire was controlled at 13 amperes and the moving speed of end effector was set to 12 millimeters per second to balance the speed of fabrication and accuracy. Duration of fabricating each foam mold block with RHWC was about 4 minutes.

After switching the end effector to the 3DCP extrusion system, coordinates of the four corners of the EPS foam printing base were recorded by manually directing the tip of the printing tozzle onto the sampled points. The maximum deviation of curved foam surface was about 5 millimeters from the original digital model design.

The curvature of the foam surface is rather smooth, allowing clay to have a better contact with the foam base; therefore, a larger nozzle with the diameter of 6 millimeter was chosen to accelerate the printing process. In addition, a double-layered path (Figure 9) design was used to minimize clay deformation under the static pressure of the concrete during casting. The water/clay ratio was determined at 35 percent empirically. Two clay parts at the height of 100 millimeters were printed on each set of foam molds, which took a total of 10 hours.

\section{CASTING, DEMOLDING AND RECYCLING}

The printed clay molds were left to cure overnight to reach a suitable strength for concrete casting. And the foam mold surface was coated with a thin layer of wet clay or vaseline as a release agent for demolding. In some cases, the surface of 3DPC parts were post-processed to smoothen the layered texture. The 3DCP parts were firmly attached to foam surfaces, and could be handled with ease; thus, the choice of casting directions was free. Hybrid molds were then set into MDF frames to complete the formwork, leaving an upturned opening for casting (Figure 9).

A high-fluidity, fast setting, off-the-shelf cement product was chosen as the base of the concrete mix for 4 of the 6 blocks. The product requires no aggregates and a water ratio of $15 \%$ to $20 \%$, and can harden within one hour. Material tests were also made with fiberglass as reinforcement. Normal portland cement based casts with sand aggregates were tested on 2 of the blocks, and left to cure for about 24 hours before demolding.

After the building blocks cured, the MDF frame and foam molds could be easily removed, leaving only the clay parts in the concrete block. There was no visible damage to the foam molds after the demolding process, and thus the foam The still humid clay molds could be easily peeled off from the blocks and recycled after mixing with water.

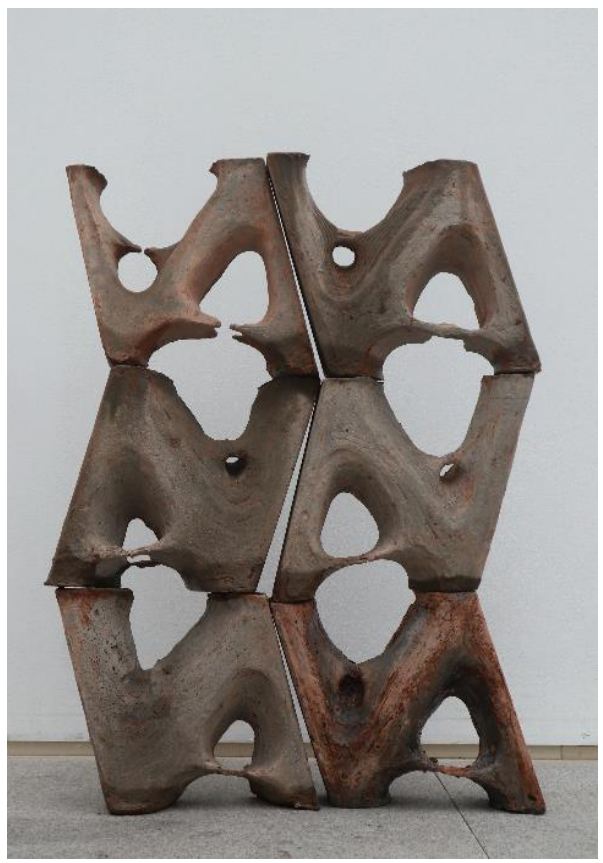

Figure 10: demonstration of the prototype tests. 


\section{TEST RESULTS}

The resultant non-standard building blocks turned out geometrically close to the original designs, especially for those cast with high-fluidity mix. However, concrete failed to form the smallest branch in 5 of the 6 blocks at the thinnest spot, implying that clay molds may have limited application in realizing smaller scale details (Figure 10).

With all mold parts being recycled or re-usable, the entire fabrication process produced almost no waste material. Empirically, the fabrication time for the hybrid formwork was comparable to, if not shorter than, that of CNC milling, and much faster than 3D printing molds of similar size. The geometries of the building blocks fabricated would be rather difficult to achieve through using RHWC alone.

\section{REFLECTION AND DISCUSSION}

This research demonstrates that the combination of subtractive and additive manufacturing enhanced through responsive computational modeling may provide a viable potential solution to the pre-fabrication of mass customized modular architectural components with balanced material and time efficiency. While the test reflects the feasibility of the proposed method as well as its potential advantages in material sustainability, geometric freedom and relative efficiency, there is still room for improvement and exploration.

Involving varied robotic fabrication techniques along with hand crafting on the same component with complex geometry often requires human intervention in-between, particularly at building component scale. This may introduce unpredictable fabrication error. In this research, the fact that the prototype building blocks were stacked with visible seams was mainly caused by the unparallel condition of the hand-crafted MDF framework. The uneven surface condition of the blocks was also caused by applying form release agent to the foam surface. How such fabrication errors could be systematically evaluated and mitigated remains a problem for hybrid formwork.

On the other hand, the integration of multiple robotic fabrication methods also challenges the directional design - fabrication workflow. Most digital fabrication processes involve the rationalization of form. Thus, each time data is transferred to and processed for the next phase of fabrication, information is inevitably deviated from the original design. That how hybrid digital fabrication should inform design of architectural geometry needs to be explored to best integrate fabrication beyond its current status as only a tool for architectural materialization.

\section{ACKNOWLEDGEMENT}

The research group would like to thank Sihan Wang from Singapore University of Technology and Design for advising on 3DCP techniques. Liyang Feng, Yating Jiang, Ningyuan Li, Zhenyan Liu, Siyu Lu, Xiaowan Xu, Yuqi Xu, and Jie Ying, who are architecture students from Zhejiang University, participated in the fabrication experiment.

\section{REFERENCES}

Bell, B., Read, T., Ede, A., \& Barnes, N. (2014). Casting nonrepetitive Geometries with Digitally Reconfigurable Surfaces. Paper presented at the 34th Annual Conference of the
Association for Computer Aided Design in Architecture (ACADIA), Los Angeles.

Brander, D., Bærentzen, J. A., Clausen, K., Fisker, A.-S., Graversen, J., Lund, M. N., .. . Søndergaard, A. (2016, 2016). Designing for hot-blade cutting: geometric approaches for high-speed manufacturing of doubly-curved architectural surfaces.

Carvalho, J. (2019). Free-form Ceramic Vault System-Taking ceramic additive manufacturing to real scale. Paper presented at the the 37th eCAADe and 23rd SIGraDi Conference.

Gardiner, J. B., \& Janssen, S. R. (2014). FreeFab. In Robotic Fabrication in Architecture, Art and Design 2014 (pp. 131146): Springer.

Jovanović, M., Raković, M., Tepavčević, B., Borovac, B., \& Nikolić, M. (2017). Robotic fabrication of freeform foam structures with quadrilateral and puzzle shaped panels. Automation in Construction, 74, 28-38.

Jovanovic, M., Vucic, M., Stulic, R., \& Petrovic, M. (2019). Design Guidelines for Zero Waste Manufacturing of Freeform EPS Facades.

Khabazi, Z., \& Budig, M. (2016). Adaptive Fabrication-Cellular Concrete Casting Using Digital Moulds. Paper presented at the Complexity \& Simplicity - the 34th eCAADe Conference.

Kontovourkis, O., Phocas, M. C., \& Katsambas, C. (2019). Digital to physical development of a reconfigurable modular formwork for concrete casting and assembling of a shell structure. Automation in Construction, 106, 102855.

Martins, P. F., de Campos, P. F., Nunes, S., \& Sousa, J. P. (2018, 2018). The Tectonics of Digitally Fabricated Concrete. A Case for Robotic Hot Wire Cutting.

Martins, P. F., \& Sousa, J. P. (2014, 10-12 September 2014). Digital fabrication technology in concrete architecture. Paper presented at the the 32nd eCAADe Conference.

Paoletti, I. (2017). Mass customization with additive manufacturing: new perspectives for multi performative building components in architecture. Paper presented at the Procedia engineering.

Schipper, H. R. (2015). Double-curved precast concrete elements: Research into technical viability of the flexible mould method.

Schipper, H. R., \& Grünewald, S. (2014, 2014). Efficient material use through smart flexible formwork method. Paper presented at the ECO-Crete: International Symposium on Environmentally Friendly Concrete, Reykjavik, Iceland.

Seibold, Z., Hinz, K., García del Castillo y López, J. L., Martínez Alonso, N., Mhatre, S., \& Bechthold, M. (2018). Ceramic Morphologies. Precision and control in paste-based additive manufacturing. Paper presented at the ACADIA 2018: Recalibration - On imprecision and Infidelity.

Søndergaard, A., Feringa, J., Nørbjerg, T., Steenstrup, K., Brander, D., Graversen, J., . . . Hattel, J. (2016). Robotic hotblade cutting. In Robotic Fabrication in Architecture, Art and Design 2016 (pp. 150-164): Springer.

Stavric, M., \& Kaftan, M. (2012). Robotic fabrication of modular formwork for non-standard concrete structures. Paper presented at the Digital Physicality -the 30th eCAADe Conference.

Timothy, W., Ena, L., Lex, R., Norman, H., Fabio, G., Matthias, K., Robert, F. (2016). Digital Concrete: Opportunities and Challenges. RILEM Technical Letters, 1(0). doi:10.21809/rilemtechlett.2016.16

Wang, S., Dritsas, S., Morel, P., \& Ho, K. (2017). Clay robotics: A hybrid 3D printing casting process. In Challenges for Technology Innovation: An Agenda for the Future (pp. 83-88): CRC Press.

Wang, S., Xuereb Conti, Z., \& Raspall, F. (2019). Optimization of Clay Mould for Concrete Casting Using Design of Experiments. 